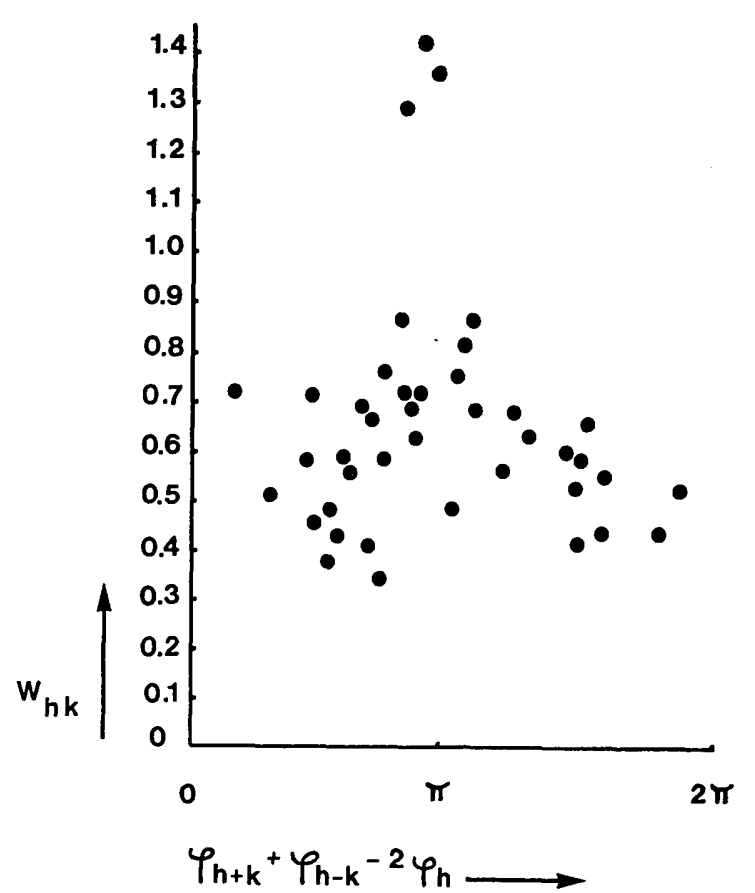

Fig. 2. The weights $W_{h k}$ are plotted as a function of $\varphi_{k+k}+$ $\varphi_{h-k}-2 \varphi_{h}$ for a hypothetical structure with 10 equal atoms in a unit cell of $P 1$ symmetry.

$h=100, h+k=110, h-k=1 \overline{1} 0$, and $k=010$ have been drawn. In Fig. 1 $(a)$ the signs of the first three reflexions are all +1 and in Fig. $1(b) S(100)=S(1 \overline{1} 0)=+1$ and $S(110)=$ -1 .

If these three reflexions are all strong then on the basis of the signs a concentration of electron density can be expected in the shaded parts in both Figures. Now it is easy to see that if refiexion 010 is strong then the situation in Fig. 1(a) is the most probable one, but if 010 is weak then the situation in Fig. $1(b)$ is the more probable. Thus Fig. 1(a) is an illustration of the $\sum_{2}$ relation and Fig. $1(b)$ of relation (3).
This geometrical explanation is not restricted to centrosymmetric structures, so that a modified relation can be proposed for non-centrosymmetric structures:

$$
\varphi_{h+k}+\varphi_{h-k}-2 \varphi_{h}=\pi
$$

for strong structure factors $h, h+k$ and $h-k$ and weak $k$. For centrosymmetric phases (4) is equivalent to (3).

This suggests the following criterion for non-centrosymmetric symmorphic space groups:

$$
\begin{aligned}
P 1 C=\sum_{h} \sum_{k} \frac{\left(\left|U_{h}\right|-\left|U_{k}\right|\right)^{2}}{\left(1-\left|U_{h+k}\right|\right)\left(1-\left|U_{h-k}\right|\right)} \\
\times\left|\pi-\left(\varphi_{h+k}+\varphi_{h-k}-2 \varphi_{h}\right)\right|
\end{aligned}
$$

in which $0 \leq \varphi_{h+k}+\varphi_{h-k}-2 \varphi_{h} \leq 2 \pi$.

The maximum value of $P 1 C$ is found for all phases $\varphi_{h}=0$ and $P 1 C$ is expected to have a minimum value for the correct phase set. For centrosymmetric phase sets $P 1 C$ is equivalent to $H K C$.

\section{Experimental test}

The $P 1 C$ criterion has been tested in two artificial structures, in which the positions of the atoms were randomly generated. For a ten-atom structure the results are $P 1 C$ (correct phases) $=8 \cdot 64$ and $P 1 C$ (all phases $=0)=26 \cdot 64$. The weights

$$
W_{h k}=\frac{\left(\left|U_{h}\right|-\left|U_{k}\right|\right)^{2}}{\left(1-\left|U_{h+k}\right|\right)\left(1-\left|U_{h-k}\right|\right)}
$$

of all terms of $P 1 C$ are plotted in Fig. 2 as a function of $\left(\varphi_{h+k}+\varphi_{h-k}-2 \varphi_{h}\right)$. This plot shows a concentration around $\pi$. For a 20-atom structure, $P 1 C$ (correct phases) $=5.85$ and $P 1 \mathrm{C}$ (all phases $=0)=15 \cdot 13$.

In our opinion the difference in $P 1 C$ values is large enough to discern the correct solution.

\section{References}

Schenk, H. \& De Jong, J. G. H. (1973). Acta Cryst. A29, 31-34.

Woolfson, M. M. (1961). Direct Methods in Crystallography, Chap. 2. Oxford: Clarendon Press.

Acta Cryst. (1973). A29, 481

Comment on The dependence of the Debye-Waller temperature factors on the atomic masses by C.Scheringer.

By P. P. M. Groenewegen* and C. Huiszoon, Chemical Physics Laboratory, Twente University of Technology, Enschede, The Netherlands

(Received 3 January 1973; accepted 27 February 1973)

The theorem on the independence of Debye-Waller $B$ values on the atomic masses in the limit of high temperatures (from which it follows that the heavier atoms in a lattice do not necessarily have the smaller $B$ values) is further discussed. It appears that the high-temperature condition prevents direct application of the theorem in its present form to molecular crystals.

\section{Introduction}

Recently Scheringer (1973), prompted by the theorem on the irrelevance of the atomic masses for Debye-Waller

* Present address: Philips Natuurkundig Laboratorium, Waalre, The Netherlands.
$B$ values in the limit of high temperatures (Huiszoon \& Groenewegen, 1972), has stressed the mass dependence of atomic mean-square amplitudes of vibration. Scheringer does not question the correctness of the derivation of the theorem; however, he argues that the theorem has been misinterpreted. We disagree with Scheringer and want to make some further remarks concerning Scheringer's point of view. 


\section{The theorem}

For zero wave vector $\mathbf{k},{ }^{*}$ all optical modes imply a fixed unit-cell centre of gravity, as Scheringer states correctly. Obviously the contributions of these modes to the atomic mean-square displacements are mass-dependent. Essential to Scheringer's argument is that this property extends to small values of the wave vector $\mathbf{k} \neq 0$. However, no mathematical proof is given for this conjecture and here we disagree. As a matter of fact from equations (1) and (15) in our earlier paper (Huiszoon \& Groenewegen, 1972) it follows that the contributions to the mean-square amplitudes of vibration become mass independent in the limit of high temperatures $\left[\hbar \omega_{j}(\mathbf{k}) / k T<1\right]$, for any value of the wave vector $\mathbf{k} \neq 0$. So for only one value of the wave vector $\mathbf{k}$, $\mathbf{k}=0$, we have a mass-dependent contribution to the DebyeWaller $B$ values, but this contribution is completely negligible when a statistical average is taken over all modes.

The theorem is not as new as we thought it to be when the derivation was presented. It has been stated before by Blackman (1956) and it is implicitly present in the work of Waller (1925) and Born (1942).

\section{Application of the theorem}

\section{Relatively simple (ionic) crystals}

By simple ionic crystals we mean any crystalline structure without molecular groups. For this type of crystal the theorem may be of practical use as the condition $T>\theta_{D}$ $\left(\simeq \hbar \omega_{\max } / k\right)$, which is necessary for the derivation, includes room-temperature experiments for most of the cases. Since experiments show that nevertheless the heavier atoms tend to have the smaller Debye-Waller $B$ values, there must be some intricate connexion between force constants and atomic numbers. In this connexion the ionic radius may be of importance [ $c f$. repulsive-potential expressions in terms of ionic radii by Pauling (1960)]. That this is a different mass dependence from the one discussed by Scheringer may be appreciated from the results of model calculations given in Table 1 . In this Table calculated $\mathrm{NaCl}$ Debye-Waller $B$ values are compared with those of a fictitious isotopic structure: force constants and lattice distance are the same for both structures; they only differ in the magnitudes of the masies of the constituent atoms (the calculations refer to the rigid-ion model discussed by

* A minor correction applies to the derivation given in our earlier paper (Huiszoon \& Groenewegen, 1972). For zero wave vector the inversion of the dynamical matrix fails because of its singularity. However, this problem occurs only for zero wave vector and hence the effect upon macroscopic properties (statistically averaged over all modes) is nill. The theorem is therefore unaffected.
Groenewegen \& Huiszoon, 1972). At high temperatures the independence of the $B$ values (of the masses) becomes apparent.

Table 1. Results of model calculations on two NaCl-type structures which differ only in the magnitudes of the masses of the constituent atoms

Force constants are the same for both compounds.

Compound I: $\mathrm{NaCl}$, atomic masses 22.98 and 35.45 . Debye temperature $375^{\circ} \mathrm{K}$. Compound II : Fictious, atomic masses 40 and 10. Debye temperature $495^{\circ} \mathrm{K}$.

\begin{tabular}{ccccc} 
& \multicolumn{4}{c}{ Debye-Waller $B$-values } \\
Temperature & Cation I & Cation II & Anion I & Anion II \\
50 & 0.46 & 0.38 & 0.34 & 0.57 \\
100 & 0.62 & 0.56 & 0.48 & 0.66 \\
300 & 1.51 & 1.49 & 1.22 & 1.30 \\
600 & 2.95 & 2.94 & 2.40 & 2.44
\end{tabular}

\section{Molecular crystals}

The internal vibrations of molecular crystals have frequencies of over $1000 \mathrm{~cm}^{-1}$. For these frequencies the condition $\hbar \omega / k T<1$ requires a temperature of over $1000^{\circ} \mathrm{K}$ from which it follows that the theorem, in the present formulation, cannot be applied to molecular crystals. In Table 1 of Scheringer's paper a number of molecular vibrational amplitudes have been collected in order to show their mass dependence. Though interesting, these data are less relevant to the present discussion. First, they have been calculated from spectroscopic data, using internal modes only, which means that the condition $\hbar \omega / k T<1$ is generally not satisfied. Second, there is only a very slight connexion with Debye-Waller $B$ values because the contribution from internal molecular vibrations to the $B$ values is usually very small, of the order of magnitude of a few percent.

We thank Professor Dr D. Feil and Dr S. Harkema for their participation in the discussion on the topic.

\section{References}

Blackman, M. (1956). Acta Cryst. 9, 734-737.

Born, M. (1942). Rep. Progr. Phys. 9, 294-333.

Groenewegen, P. P. M. \& Huiszoon, C. (1972). Acta Cryst. A 28, 166-169.

Huiszoon, C. \& Groenewegen, P. P. M. (1972). Acta Cryst. A 28, 170-172.

PAuling, L. (1960). The Nature of the Chemical Bond, p.524. Ithaca: Cornell Univ. Press.

SCheringer, C. (1973). Acta Cryst. A 29, 82-86.

WALLER, I. (1925). Thesis (Uppsala). 